\title{
Influencia del contexto institucional en el trabajo de los profesores de matemáticas en la educación superior técnico-profesional en Chile*
}

\author{
Influence of the institutional context on mathematics teachers' work \\ in technical and vocational higher education in Chile
}

\author{
Felipe Droguett, Sergio Celis \\ Escuela de Ingeniería y Ciencias, FCFM, Universidad de Chile \\ Correo electrónico: scelis@ing.uchile.cl
}

\begin{abstract}
RESUMEN
La tasa de deserción en primer año del sistema de educación superior técnico profesional (ESTP) en Chile es superior al 30\%. Una de las principales razones académicas para el abandono es el aprendizaje y enseñanza de las matemáticas. Las instituciones entienden esto y proponen distintas políticas para mejorar estos indicadores. Sin embargo, es escasa la investigación sobre el efecto de estas iniciativas en los profesores de matemáticas en general. Este artículo busca entender la relación entre el contexto institucional y lo que sucede con los profesores de matemática en la ESTP, usando la perspectiva de las obligaciones profesionales y del hacer sentido. Mediante entrevistas y grupos de discusión de directivos y profesores, concluimos que las políticas institucionales no están pensadas para apoyar el trabajo del docente y en ocasiones traban un buen trabajo en aula. Este artículo discute estos resultados y plantea recomendaciones para el conjunto del sistema de ESTP.
\end{abstract}

Palabras claves: enseñanza de la matemática, obligaciones profesionales, política institucional

\begin{abstract}
First year dropout rates in the system of technical and vocational higher education (ESTP) in Chile is greater than $30 \%$. One of the main reasons of this rate is the teaching and learning of mathematics. Institutions understand this and promote different policy in order to improve this indicator. However, there is little research on the effect of these initiatives on mathematics teachers in general. This article seeks to understand the relationship between the institutional context and what happens with mathematics teachers in ESTP, using the perspectives of professional obligations and sensemaking. Through interviews and group discussions with administrators and teachers, we found that institutional policy is not designed to support teachers' work and sometimes obstruct good classroom work. We discuss implications and recommendation for the whole ESTP system.
\end{abstract}

Key words: mathematics education, professional duties, institutional policy 
Estudios Pedagógicos XLIV, N $^{\circ}$ 3: 235-252, 2018

INFLUENCIA DEL CONTEXTO INSTITUCIONAL EN EL TRABAJO DE LOS PROFESORES DE MATEMÁTICAS EN LA EDUCACIÓN SUPERIOR TÉCNICO-PROFESIONAL EN CHILE

\section{INTRODUCCIÓN}

Aunque desde el 2011 la educación ha acaparado la discusión pública y académica, el debate se centrado en la educación escolar y universitaria, relegando a segundo plano lo que ocurre en la educación superior técnico profesional (ESTP). Es necesario analizar lo que sucede en la ESTP porque concentra más de la mitad de la matrícula de primer año en el sistema de educación superior y porque recibe a los estudiantes de los sectores socioeconómicos más bajos (Espejo, 2016). Más importante aún, aquellos que egresan de la ESTP experimentan un importante ascenso social, incluso mayor al de algunas carreras universitarias (MINEDUC, 2012). Sin embargo, es también en la ESTP donde se alcanzan las tasas más altas de deserción del sistema (SIES, 2016a). La masividad e impacto del sector requiere mayor investigación para entender las dinámicas y mecanismos de éxito, en especial con un foco en las salas de clases, en donde cientos de miles de estudiantes ven aumentar o frustrar sus expectativas de futuro. La presente investigación aborda las problemáticas de la ESTP desde la mirada de los profesores de matemáticas, aquellos que tienen contacto con la mayor cantidad de estudiantes y de múltiples especialidades. El quehacer de los profesores y su relación con la institución es un factor crítico para entender cómo se desarrolla el proceso de enseñanza y aprendizaje en la ESTP, el cual ha estado poco estudiado en Chile.

Si se comprende cómo los profesores hacen sentido de su contexto institucional en su práctica pedagógica, es posible entender de mejor manera el impacto que tienen las políticas institucionales y nacionales en el aula. Las consecuencias de comprender esta relación entre profesores y su contexto son significativas: políticas mejor diseñadas y con mayor capacidad de implementación efectiva, políticas institucionales que fortalezcan la capacidad de agencia de los profesores y en último término, mayor calidad en el sistema de ESTP.

\section{REVISIÓN DE LA LITERATURA}

\subsection{SISTEMA DE ESTP EN CHILE}

La ESTP, así como el conjunto del sistema de educación superior en Chile, se edificó a partir de la reforma educacional de 1981. Esta reforma dividió las instituciones de educación superior en tres categorías: universidades; institutos profesionales (IP) que entregan títulos técnicos de nivel superior y títulos profesionales no conducentes a grado académico y centros de formación técnica (CFT) que sólo entregan títulos técnicos. Una de las características de la reforma es que mientras a las universidades se les impidió formalmente el lucro, esta prohibición no se expresó en la ESTP. Esto sumado a la nula oferta pública con que quedaba este sector, hizo que el proceso de privatización fuera mucho más intenso en la ESTP (Sanhueza, 2015).

Desde la década de 1980 la matrícula total del sistema de educación superior aumentó, llegando en la actualidad a niveles de cobertura similares al promedio de la OCDE (Paredes \& Sevilla, 2017), los cuales se deben en gran medida al explosivo aumento de la matrícula de ESTP a partir de las modificaciones en el financiamiento estudiantil ocurridas durante la primera década del siglo XXI. Las altas tasas de crecimiento de la matrícula de ESTP 
en comparación al crecimiento del conjunto de la matrícula de Educación Superior, le permitieron a este subsistema en una década (2000-2010) aumentar de 133 mil estudiantes a 352 mil (SIES, 2018a), llegando a concentrar a más del 43\% de la matrícula de pregrado y al 57\% de la matrícula de primer año (SIES, 2014). En 2018, hay cerca de 510 mil estudiantes matriculados en la ESTP (SIES, 2018b).

Cabe destacar que gran parte de este crecimiento de matrícula se ha concentrado en los sectores socioeconómicos más vulnerables. Según los datos de la encuesta CASEN 2015, mientras que en el subsistema universitario el 46,3\% son estudiantes de los tres primeros quintiles, en el caso de la ESTP esta cifra llega al 60,3\%.

Tabla 1. Distribución de la matrícula (en \%) de educación superior por tipo de institución de educación superior y quintil de ingresos (2015)

\begin{tabular}{|l|c|c|c|c|c|c|}
\hline \multirow{2}{*}{ Tipo institución } & \multicolumn{5}{|c|}{ Quintiles } & \multirow{2}{*}{ Total } \\
\cline { 2 - 7 } & $1 \mathrm{Q}$ & $2 \mathrm{Q}$ & $3 \mathrm{Q}$ & $4 \mathrm{Q}$ & $5 \mathrm{Q}$ & \\
\hline Universidad & 8,6 & 16,0 & 21,7 & 25,6 & 28,1 & 100 \\
\hline CFT e IP & 10,1 & 22,7 & 27,4 & 26,7 & 13 & 100 \\
\hline \multicolumn{1}{|c|}{ Total } & 9,1 & 18,4 & 23,7 & 26 & 22,8 & 100 \\
\hline
\end{tabular}

Fuente: elaboración propia a partir de datos CASEN 2015

No es mucho lo que se puede decir en relación a los profesores de estas instituciones, dada la escaza investigación en torno a ellos. De acuerdo a los datos administrativos del MINEDUC es posible plantear que los profesores de CFT e IP presentan bajos estándares de certificación académica (MINEDUC, 2012); están contratados por pocas horas y trabajan en más de una institución (CNED, 2017), las cuales además presentan una relación estudiantes por profesor mayor que las universidades (CNED, 2017).

\subsection{ENSEÑANZA DE LA MATEMÁTICA EN LA ESTP}

Entender cómo afecta el contexto institucional en las prácticas de los profesores de la ESTP resulta particularmente significativo para la enseñanza de las matemáticas. En muchos programas las matemáticas funcionan como gatekeeper (o portero en español) para la continuidad de los estudios, y diversas investigaciones han relevado la importancia de las matemáticas en indicadores críticos tales como el acceso, la permanencia y el éxito académico de los estudiantes en educación superior (Kim, Kim, DesJardins \& McCall, 2015; Melguizo, Kosiewicz, Prather \& Bos, 2014; Hodara \& Jaggars, 2014), sobre todo en carreras relacionadas a las ciencias y tecnología (Blair, Kirkman \& Maxwell, 2013).

En la ESTP uno de los principales temas de preocupación, sino el principal, es la retención de los estudiantes de primer año, el cual llega a un $66 \%$ en caso de los IP y de $68 \%$ en el caso de los CFT, cifras aproximadamente un 10\% más bajas que en el subsistema universitario (SIES, 2016b). De ahí también que estudiar la enseñanza de matemáticas 
cobre mayor importancia en estas instituciones. Cabe destacar además, que por el número de estudiantes y de cursos, la enseñanza de las matemáticas tiene un alto peso relativo en el sistema. Sólo en las áreas de tecnología y administración y comercio -áreas en dónde las matemáticas juegan un rol fundamental- se concentra un $64 \%$ de la matrícula de las IES de la ESTP.

Figura 1: Matrícula total (\%) en CFT e IP por área de conocimiento (2016)

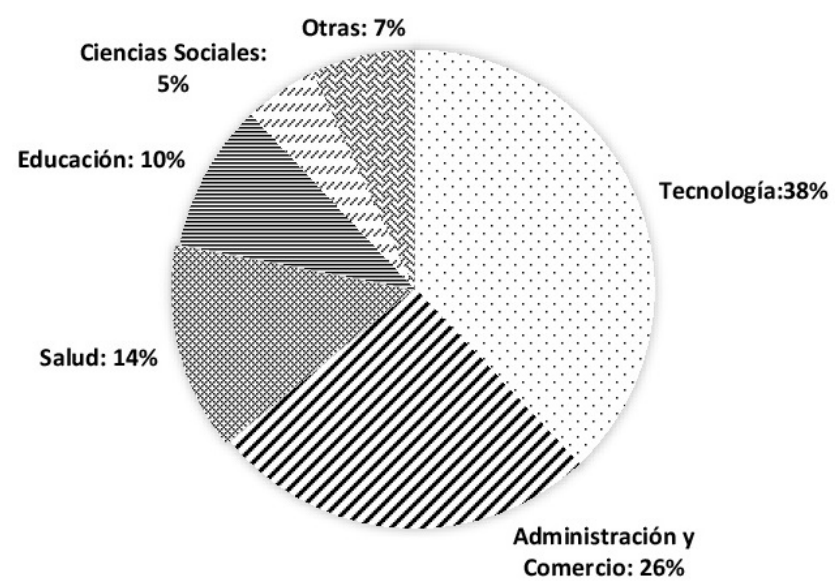

Fuente: elaboración propia a partir de datos ÍNDICES - CNED (2017)

\subsection{ENFOQUE MULTIDIMENSIONAL DE LA ENSEÑANZA EN LA EDUCACIÓN SUPERIOR}

Las investigaciones sobre las aulas en educación superior apuntan crecientemente a incorporar dimensiones sociales e institucionales, entendiendo la enseñanza como una actividad compleja y multidimensional en dónde tanto el contexto como las interacciones sociales dentro del aula juegan un papel fundamental (Hora \& Ferrare, 2013; Lattuca \& Stark, 2009). Lo que indican estas investigaciones es que comprender el contexto en el cual se desarrolla el trabajo docente permite entender el porqué de las decisiones de los profesores y por lo tanto, entrega pistas sobre el porqué de ciertos resultados educativos. Este enfoque asume que la racionalidad de los profesores se encuentra enmarcada en un conjunto de obligaciones que no sólo tienen que ver con su conocimiento sobre la materia que está impartiendo o el punto de vista disciplinar desde el cuál se sitúa, sino que también por obligaciones con la institución en la que está trabajando, con el grupo de estudiantes a los que les está enseñando y con cada uno de esos estudiantes de forma individual (Herbst \& Chazan, 2011).

La investigación sobre las matemáticas en el sistema de educación escolar es nutrida y permite una aproximación más precisa al fenómeno de la educación superior. Diversas investigaciones han dado cuenta de la importancia de las interacciones sociales y procesos individuales en la forma en que se enseña y se aprende matemáticas (Sfard, 2001; Yackel \& Cobb, 1996); del impacto que tiene en el aprendizaje las interacciones que ocurren 
dentro de la sala de clases (Kunter \& Voss, 2013) y de la relación entre el aprendizaje de los estudiantes con el conocimiento disciplinar de los profesores (Ball, Lubienski \& Mewborn, 2001; Hill, Ball \& Schilling, 2008) y la forma en que estos toman decisiones (Mason, 2016; Schoenfeld, 2016). En particular para este estudio, revisten importancia las recientes investigaciones sobre la enseñanza de la matemática en los community colleges norteamericanos (Lande \& Mesa, 2016; Mesa, Celis \& Lande, 2014), los cuales por sus características son posibles de asimilar a las instituciones de la ESTP chilena. Dichas investigaciones han confirmado que también en estas instituciones, los contextos institucionales y las interacciones sociales tienen una relevancia en lo que realizan las y los profesores en las salas de clases (Lande \& Mesa, 2016).

\section{MARCO CONCEPTUAL}

\subsection{EL CONTEXTO INSTITUCIONAL DE LA ENSEÑANZA Y LAS OBLIGACIONES PROFESIONALES}

Las investigaciones sobre los procesos de enseñanza en educación superior no han puesto en el centro el estudio sobre los contextos sociales e institucionales en los cuales se inscriben dichos procesos. Aun cuando los nuevos enfoques en relación a la investigación incorporan aspectos que anteriormente no tenían mucha preponderancia -como los procesos de aprendizaje (Leindhardt \& Greeno, 1986; Leindhardt, 1989; Schoenfeld, 1985) y el contenido específico de la enseñanza de matemáticas (Carpenter, Fennema, Franke, Levi \& Empson, 1999; Carpenter, Fennema \& Franke, 1996)- la incorporación del contexto todavía aparece ajena. Steinbring (2005) plantea que la investigación sobre la enseñanza de matemáticas se ha circunscrito a la investigación de las relaciones entre estudiantes y de estos con sus profesores. El ambiente de la enseñanza sin embargo, no ha sido tratado con la misma intensidad (Chazan, Herbst \& Clark, 2016). El insuficiente desarrollo de investigaciones que den cuenta del contexto, la creciente incorporación de factores culturales o contextuales a las investigaciones sobre la enseñanza de matemáticas y la evidencia que da cuenta de la importancia de estos factores en el proceso educativo, hace que sea necesario poner más atención en el contexto social e institucional.

La propuesta teórica de Chazan et al. (2016) que da cuenta del contexto en el cual se desarrolla la actividad educativa comienza por la identificación de diversos actores claves o stakeholders presentes en el proceso de instrucción. El actuar y las preocupaciones de los stakeholders derivan en obligaciones para los profesores en el proceso educativo. Este conjunto de obligaciones explícitas e implícitas, Chazan et al. (2016) las denominan como obligaciones profesionales. Para los autores, es posible definir este concepto como "los valores y las normas que son obligatorias por la fuerza de la costumbre (...) y refieren específicamente a cómo los ambientes limitan y condicionan la posición del profesor de matemáticas" (Chazan et al., 2016: 1063, traducción propia).

Los tipos de obligaciones profesionales hacen referencia al tipo de stakeholder interesado en el proceso educativo. Chazan et al. (2016) plantean que existen cuatro tipos de obligaciones profesionales sobre docentes: a) con la disciplina, que se puede manifestar la revisión y actualización de los contenidos, tareas, libros de textos y tecnologías utilizadas en el proceso de enseñanza;. b) con el estudiante como individuo, la cual implica asumir la posición de proveedor de un servicio que tiene que satisfacer los requerimientos del 
Estudios Pedagógicos XLIV, N 3: 235-252, 2018

INFLUENCIA DEL CONTEXTO INSTITUCIONAL EN EL TRABAJO DE LOS PROFESORES DE MATEMÁTICAS EN LA EDUCACIÓN SUPERIOR TÉCNICO-PROFESIONAL EN CHILE

cliente; c) con la sociedad, obligación que hace referencia a los resultados de la enseñanza en términos de aporte a la comunidad, la cultura, la economía, el cumplimiento de las leyes, la cohesión social, entre otras cosas y d) con la institución. Para Chazan y Herbst (2012) las obligaciones con la institución buscan que la enseñanza de matemáticas se produzca en base a criterios de legalidad, eficiencia y eficacia. Una de las particularidades que tendrían las obligaciones institucionales respecto al resto de las obligaciones profesionales en las cuales se encuentra situado el trabajo docente, es que el foco de ellas está puesto tanto en los resultados de la enseñanza como en el proceso (Chazan et al., 2016).

El sistema educacional y las instituciones que lo componen tienen la misión de resguardar el cumplimiento de los objetivos institucionales dentro de la sala de clases, para lo cual hacen uso de distintos instrumentos que se transforman en obligaciones explícitas (Chazan et al., 2016). Sin embargo, y al igual que con el resto de las obligaciones, existen obligaciones implícitas que no son regla, pero que de todas maneras limitan el campo de accion de las y los docentes. Un ejemplo de ello es que por muy bajo que sea nivel académico de los estudiantes, no es posible reprobar a todo su curso por las consecuencias institucionales que dicha acción tendría. Otras obligaciones institucionales que determinan de forma implícita el trabajo docente son por ejemplo el calendario académico, los horarios de trabajo y de clases, la variedad de ofertas de cursos de matemáticas y las políticas de evaluación de la institución (Chazan et al., 2016).

Otra categoría que puede entenderse como parte de las obligaciones institucionales es el acceso a recursos profesionales por parte de los profesores, entendidos estos como los recursos materiales y tecnológicos con el que las y los docentes cuentan para llevar a cabo la instrucción, así como los recursos sociales e intelectuales que poseen (Cohen, Raudenbush $\&$ Ball, 2003). El acceso a mayores o menores recursos y la preferencia por el uso de algunos en desmedro de otros, depende de las trayectorias y las características individuales de los profesores, pero por otro lado, es el contexto institucional el que provee otro tipo de recursos y manifiesta ciertas preferencias por el uso de ellos (Chazan et al., 2016).

\subsection{EL HACER SENTIDO DEL CONTEXTO INSTITUCIONAL}

El estudio de la influencia de las políticas públicas e institucionales en los profesores de enseñanza primaria es de larga data. En particular, desde los años 1970s en adelante, ha primado el argumento del desacoplamiento (decoupling) entre docentes y su entorno institucional para entender por qué los cambios impulsados desde la política no logran implementarse en el aula (Coburn, 2004). En términos generales la teoría del desacoplamiento plantea que en las escuelas los profesores responden ante las presiones y obligaciones institucionales a través de modificaciones simbólicas de las prácticas ya realizadas, no logrando realizar cambios que respondan al centro de lo que las políticas del entorno están apuntando (Deal \& Celloti, 1980; Driscoll, 1995; Malen, Oganawa \& Kranz, 1990; Meyer \& Rowan, 1977; Meyer \& Rowan, 1978). La prevalencia de la teoría del desacoplamiento ha contribuido a reducir el interés por investigar directamente la relación entre el contexto institucional y el trabajo de las y los profesores (Rowan \& Miskel, 1999), centrándose más bien los esfuerzos en investigar la relación entre las instituciones y las modificaciones en las políticas educativas exógenas (Malen et al. 1990; Malen \& Ogawa, 1988).

A partir de los esfuerzos por desarrollar conceptos diferentes a la teoría del desacoplamiento (Metz, 1989; Cuban, 1984; EEPA, 1990; Talbert \& McLaughlin, 1994) 
es que se incorpora el concepto de hacer sentido (o sensemaking en inglés). Siguiendo la definición de Berger y Luckmann (1966), la teoría de hacer sentido plantea que la forma en que las cosas son, la forma en que las cosas deberían ser, y la forma en que las cosas son hechas, son construcciones sociales e históricas a través de interacciones interpersonales. En palabras de Weick, Sutcliffe \& Obstfeld, hacer sentido implica "el desarrollo retrospectivo en el presente, de imágenes plausibles que racionalizan lo que la gente está haciendo", lo que significa que éste puede entenderse "como un proceso significativo de organización" (Weick, Sutcliffe \& Obstfeld, 2005: 409). Así, la acción individual se basa en cómo las personas seleccionan información desde el entorno, entregándole significados a dicha información y actuando en función de esa interpretación (Weick, 1995). La selección e interpretación de la información contextual se hace a partir de las diferentes estructuras de pensamiento o visiones de mundo que poseen los sujetos (Weick, 1995).

Ahora bien, ¿cuál es el impacto que tiene el concepto de hacer sentido en la relación de las y los profesores con su entorno? Según Coburn (2004) este concepto permite reconceptualizar la relación entre el entorno institucional y lo que ocurre en la sala de clases. Según esta autora, los profesores realizan una mediación activa de los mensajes sobre la enseñanza que encuentran en el entorno, teniendo estos por tanto, una influencia en la sala de clases mediada por la prácticas y visiones de mundo preexistentes de las y los docentes. El desacoplamiento por tanto, se entendería como una forma más en la cual los profesores reaccionan ante estímulos y obligaciones del contexto institucional.

\subsection{PREGUNTA DE INVESTIGACIÓN}

En base a los antecedentes anteriormente descritos y la conceptualización sobre obligaciones institucionales y el hacer sentido, es que se propone la siguiente pregunta de investigación: ¿Cómo las obligaciones institucionales influyen en el trabajo de los profesores de matemáticas de las instituciones de ESTP en Chile? Para responder a dicha pregunta, se trabajará en base a dos objetivos: a) describir las obligaciones institucionales que influyen en el trabajo de las y los profesores de matemáticas en la ESTP y b) identificar cómo las y los profesores de matemáticas hacen sentido de las obligaciones institucionales recibidas desde el contexto institucional.

\section{METODOLOGÍA}

\subsection{MUESTRA Y RECOLECCIÓN DE DATOS}

Para describir las obligaciones profesionales en las cuales se enmarca el trabajo de los profesores de la ESTP se realizó un análisis de entrevistas semi-estructuradas (Merriam, 2009) y grupos focales de discusión (Horn \& Kane, 2015) realizados a actores claves en el sistema en dónde se les consultó de las percepciones sobre sus estudiantes y las prácticas, concepciones e influencias de la enseñanza en ESTP. Los participantes de estas entrevistas fueron 8 directivos de instituciones de la ESTP, 13 profesores de matemáticas de un establecimiento y 4 expertos del MINEDUC y de organizaciones vinculados a la ESTP.

Para las entrevistas semiestructuradas a directivos, se seleccionaron las instituciones con mayor matrícula: AIEP, DUOC, INACAP y Santo Tomás, los cuales en conjunto 
Estudios Pedagógicos XLIV, $\mathrm{N}^{\circ}$ 3: 235-252, 2018

INFLUENCIA DEL CONTEXTO INSTITUCIONAL EN EL TRABAJO DE LOS PROFESORES DE MATEMÁTICAS EN LA EDUCACIÓN SUPERIOR TÉCNICO-PROFESIONAL EN CHILE

representan a un 77\% del total de la matrícula de CFT e IP el 2017 (Menéndez, 2017). Entre estos directivos se incluyen vicerrectores académicos, y directores y coordinadores generales de ciencias básicas, aseguramiento de la calidad, y pedagogías. Entre los entrevistados hay 5 mujeres y 3 hombres.

La forma de identificar cómo los docentes hacen sentido respecto al contexto institucional fue a través de 5 entrevistas semiestructuradas y de sesiones de discusiones grupales basadas en análisis de video, en las cuales participaron otros 8 profesores. Para entender con mayor profundidad las relaciones del contexto institucional con el aula, la muestra estuvo limitada a profesores de matemáticas de una sola institución. Esta institución cumple con las características de tener matrícula tanto de CFT como de IP, en la cual se imparten cursos de matemáticas para la mayoría de sus especialidades. La institución escogida tiene una alta matrícula (más de 20.000 estudiantes) y posee diversas sedes en todo el país. En promedio estos profesores tienen 11.5 años enseñando en la ESTP, en un rango de 3 a 30 años. En su mayoría cuentan con el grado licenciatura en pedagogía y cuatro de ellos además tiene posgrados en educación o en áreas de las ingenierías y ciencias. En la muestra de profesores hay 5 mujeres y 8 hombres.

Las discusiones grupales basadas en análisis de video se dieron en el marco de un programa de desarrollo profesional (PDP) anual basado en un aprendizaje activo de la matemática. Una de las líneas de acción del PDP fue realizar pequeños grupos de discusión (de tres a cuatro profesores) de videos con episodios de los mismos profesores interactuando con sus estudiantes en el aula. En promedio cada sesión tuvo una duración de 90 minutos. Estos grupos de discusión fueron facilitados por profesionales del PDP y entregaron información respecto a cómo las distintas obligaciones -entre ellas las institucionales- afectaban a los profesores y los llevaban a tomar distintas decisiones en el aula. Estos grupos de discusión basados en video permiten que los profesores conversen sobre aquellas normas implícitas del trabajo del aula, en referencia al concepto de obligaciones profesionales (Chazan et al., 2016). El nutrirse de episodios en los cuales los profesores intentan implementar metodologías innovadoras para ellos, acentúa al discusión sobre lo qué es y no posible o recomendable en el aula, material esencial para el análisis de obligaciones profesionales y del hacer sentido. En total, se analizaron ocho grupos de discusión basados en el análisis de videos.

\subsection{ANÁLISIS DE DATOS}

Todas las entrevistas semi-estructuradas y los grupos de discusión fueron transcritos verbatin. Luego, se realizó un análisis semi-deductivo (Merriam, 2009) donde primero se definieron las categorías en base al marco conceptual y luego se analizaron temáticamente (Braun \& Clarke, 2012) nuevos conceptos y subcategorías. Primero, se definieron dos conceptos centrales que responden a la pregunta de investigación: Obligaciones institucionales y formas de hacer sentido de los profesores. En base a Chazan et al. (2016) se entenderán las obligaciones institucionales como los valores y las normas emanados de la institución o su contexto que, referidos a la búsqueda de legalidad, eficiencia y eficacia del proceso educativo, son obligatorias por la fuerza de la costumbre y limitan y condicionan la posición de las y los profesores de matemáticas. El segundo concepto operacionalizado fue el análisis de las Formas de hacer sentido de los profesores. En base al trabajo de Weick et al. (2005) y Coburn (2004) se entenderá como Formas de hacer sentido las 
distintas maneras que tienen las y los docentes para procesar los mensajes de su contexto y adecuarlo a su trabajo.

A través de diferentes dimensiones y sub-dimensiones de estos conceptos es que se fue codificando la información (entrevistas y grupos de discusión), separando primero aquellos elementos referentes a las obligaciones institucionales con respecto al resto. Aquellos textos referentes a obligaciones fueron luego agrupados temáticamente. Cada autor analizó el material de manera independiente y luego se reunieron a discutir los resultados emergentes y a dirimir sobre divergencias. Para este proceso de análisis se usó el programa Nvivo 11 v4.1.

\section{RESULTADOS}

Antes de presentar aquellos resultados que responden a la pregunta de investigación, discutimos brevemente el perfil de los profesores de matemáticas según los directivos y los profesores participantes de este estudio. Esto es necesario dada la escaza investigación que existe sobre estos docentes.

\section{1. ¿QUIÉNES SON LOS PROFESORES DE MATEMÁTICAS DE LA ESTP?}

El perfil de los profesores matemáticas, al igual que para quienes realizan clases en el ciclo básico en la ESTP, es diferente al de los docentes de especialidad ${ }^{1}$. Cabe destacar, que los profesores de ciclo básico agrupan a parte importante de profesores de la ESTP. Sólo la institución focalizada tiene más de 300 profesores de matemáticas en todas sus sedes. Los profesores de ciclo básico están mucho más vinculados a la docencia que sus pares de especialidad. Por ejemplo, en matemáticas, la mayoría de los profesores son licenciados en pedagogía matemática, aunque también hay quienes estudiaron licenciatura en matemáticas e ingeniería de distintas áreas. Además, en la mayoría de los casos, la actividad principal del docente es la docencia. En contraste, los profesores de especialidad suelen trabajar en empresas privadas en dónde el valor/hora de su trabajo es mucho mayor al que ofrecen las instituciones, lo cual dificulta la contratación de estos. En el caso de los profesores de matemáticas, la competencia por salarios se da con respecto a otras instituciones educacionales de enseñanza media o universitarias.

Los profesores de matemáticas de la ESTP están alejados de la realidad de sus pares universitarios. De partida, son muy pocos quienes tienen un grado académico mayor a una licenciatura o título profesional. Aun cuando presenten importantes diferencias con sus pares de enseñanza media, los profesores de la ESTP tienen características que lo asemejan mucho más-por su perfil, por las tareas que realizan y los contenidos que exponen-a un docente de colegio a que académico universitario. Además, la mayoría realiza clases en liceos y colegios antes de ingresar a la educación superior. Para la mayoría de nuestros entrevistados, la experiencia de enseñar en la ESTP supera a la de establecimientos de enseñanza media debido en gran parte, a la motivación e interés que los estudiantes tienen por aprender. Así, quienes llegan a hacer clases a CFT e IP en muchos casos lo hacen

Los ramos de ciclo básico son de formación general y se dan para estudiantes de distintas especialidades. Ejemplos de estos ramos son los de matemáticas o inglés. Los ramos de especialidad por otro lado, son los propios de cada especialidad y requieren profesores expertos en esa área en particular, tales como contabilidad, mecánica automotriz, administración en hotelería y servicios, o prevención de riesgos. 
Estudios Pedagógicos XLIV, N $^{\circ}$ 3: 235-252, 2018

INFLUENCIA DEL CONTEXTO INSTITUCIONAL EN EL TRABAJO DE LOS PROFESORES DE MATEMÁTICAS EN LA EDUCACIÓN SUPERIOR TÉCNICO-PROFESIONAL EN CHILE

"escapando" del sistema escolar en dónde los estudiantes no muestran interés y motivación sobre todo con las matemáticas. A pesar de las posibles carencias académicas, quienes se matriculan en la ESTP lo hacen con la claridad de que necesitan el título de la institución para ingresar (o reinsertarse) rápidamente al mundo laboral. Lo anterior hace que tengan una disposición diferente para enfrentar las clases, lo cual, para quien viene de hacer clases un colegio, se transforma en un aliciente para continuar trabajando en la institución.

La diferencia fundamental de la educación superior con la educación secundaria es que el chico que está en la educación superior tiene un objetivo súper, súper claro y transparente. Tiene un objetivo y ese objetivo hace que las disciplinas con las cuales se encuentra, él las va a tomar mucho más en serio (Profesor, 15 años de experiencia).

Las ganas de aprender de los estudiantes parecieran ser el factor común para entender la motivación docente para realizar clases en la ESTP en vez de trabajar en la educación escolar. Pero a la vez la motivación de los estudiantes, implica que quien les enseña debe asumir un mayor compromiso que requiere necesariamente tener vocación para enseñar. Según los docentes, este compromiso no solo es un compromiso pedagógico sino que también afectivo. Debe ser un compromiso por apoyarles, entregarles seguridad y cariño a estudiantes que muy probablemente durante toda su vida escolar tuvieron problemas y frustraciones con las matemáticas.

Vienen con mucha desconfianza de sus capacidades. Yo creo que vienen con inseguridades, con miedos de "yo no aprendí nunca matemática por qué voy a aprender ahora. No aprendí matemática hace 10 años atrás por qué voy a aprender ahora”. Ellos vienen con mucha inseguridad, yo creo que eso es lo que más necesitan. Suena un poco como quizás... como cariño y afecto (Profesor, 3 años de experiencia).

En cuanto a las condiciones laborales, la mayoría de los profesores están contratados a régimen parcial, es decir, en función de las horas de clases y los cursos que pueden atender. Lo anterior, deriva en que para completar una jornada completa, los profesores tengan que trabajar en más de una institución, ya sea en colegios o en otra institución de educación superior. Este diagnóstico se condice con los datos que brinda el CNED y que indican que sólo un $11 \%$ y un $7 \%$ de las y los docentes en IP y CFT respectivamente, tienen un contrato de más de 39 horas en su institución (CNED, 2017). La forma de fidelización de los profesores por parte de la institución es el contrato indefinido. La cantidad de horas del contrato no varía, sin embargo se le entrega la estabilidad de un contrato indefinido a quienes cumplen con una cantidad de tiempo en la institución y sean evaluados de buena forma.

Un gran incentivo para un docente nuevo es, después de hacer una buena docencia y tener una buena evaluación docente, sabe, de alguna forma, que después de su segundo contrato fijo va a venir un contrato indefinido con un número de horas específico (Directivo ESTP).

Esta realidad contractual también tiene consecuencias en el trabajo de los profesores y el tiempo que pueden dedicar a realizar actividades no lectivas. Pese a que en la ESTP los profesores de matemáticas no desarrollan investigación, sí necesitan importantes horas para 
planificar clases, construir problemas, y planificar formas de apoyar a los alumnos fuera de la sala de clases. De cierto modo, sin horas disponibles, no hay planificación de clases, sino que una repetición mecánica de la planificación que viene de la institución, no existiendo adaptación de los contenidos al ritmo de los estudiantes y tampoco hay apoyo pedagógico a los estudiantes salvo a través de los programas de apoyo institucional o cuando hay "buena voluntad" del profesor.

Hay un desgaste súper grande y aquí mismo pasa, nosotros no tenemos horas para poder atender a los alumnos fuera del horario de clases y si lo hacemos es por amor al arte. Tenemos que planificar, hacer material, un montón de cosas, que no están incluidas dentro de tu horario de trabajo. Entonces tú no tienes espacio ¿cómo voy a hacer yo una buena clase si no tengo espacio para sentarme a crear, sentarme a conversar? (Profesora, 10 años de experiencia).

Otro de los elementos que destacan en relación a las condiciones contractuales de las y los docentes de matemáticas en CFT e IP, es que son contratados por áreas siendo que realizan clases para estudiantes de varias especialidades. Por ejemplo, quien ingresa a trabajar a una institución puede ser contratado por el área de mecánica aunque haga clases en mecánica, informática y en administración, mientras que otro de sus colegas puede estar contratado por otra área haciéndoles cursos a los mismos estudiantes. Lo anterior tiene consecuencias en la identidad y sentido de pertenencia de las y los profesores con su disciplina. Hay pocos espacios compartidos entre ellos, y es escaso el desarrollo de una identidad común:

Cuando yo recién llegué empecé a traer unos logos de "profesor de matemática". Te generaba una identidad, ¿sí? cuando llegué empezaron a mirar mal y ahí dije "no, esto no va por este lado" (...) No hay una identidad de los profesores de matemática. (...) nosotros trabajamos en área pedagógica [matemática], pero nuestro contrato es desde las áreas, desde mecánica, desde minería, desde informática, desde construcción (Profesor, 15 años de experiencia).

\subsection{ENSEÑANZA CONTRA EL TIEMPO Y LEJANÍA DEL "OLIMPO"}

Para los profesores, la institución es vista como un ente alejado de la sala de clases: una institución que mandata una construcción curricular que muchas veces no responde a la realidad de la sala de clases y a la posibilidad de que se lleve a cabo un efectivo proceso pedagógico. La obligación por el currículum y direcciones institucionales genera una crítica en los profesores, quienes cuestionan la capacidad que tienen las autoridades centrales por recoger sus opiniones como profesores -quienes tienen "los pies en el barro"- a la hora de definir los estándares de conocimiento que se exigen por curso. Los profesores sienten que lo que les pide la institución es algo formal y vacío en términos de contenido. Que se hagan las clases bien y que tengan buena evaluación, pero nada más. Poco en términos de aprendizaje del estudiante.

[El Departamento de Currículo] en el más allá sentado en el olimpo nunca se ha hecho presente en decir "Chicos, ¿qué les parece este [resultado de aprendizaje]? ¿Está bien 
Estudios Pedagógicos XLIV, $\mathrm{N}^{\circ}$ 3: 235-252, 2018

INFLUENCIA DEL CONTEXTO INSTITUCIONAL EN EL TRABAJO DE LOS PROFESORES DE MATEMÁTICAS EN LA EDUCACIÓN SUPERIOR TÉCNICO-PROFESIONAL EN CHILE

diseñado? ¿Tiene estructura? ¿Está correlacionado? ¿Alimenta a las otras asignaturas que vienen?" Uno sabe lo que está pasando y no te escuchan. No hay participación, por ejemplo, en los [resultados de aprendizaje] de las asignaturas. Los descriptores están súper mal diseñados en sus tiempos, en sus formas, en su fondo. Y lo hemos dicho, muchos, todos. Y no baja nadie de casa central a escuchar la opinión de los profes (Profesora, 10 años de experiencia).

Por otro lado, el uso de los tiempos es una de las características administrativas que más afecta el trabajo de los profesores. Las instituciones de la ESTP tienen por objetivo entregar un título técnico o profesional lo antes posible, puesto que su relación con el mundo laboral es mucho más directa que en el mundo universitario. En palabras de una directiva: "en la ESTP estamos todos contra el tiempo". La exigencia por usar los tiempos de manera eficiente está patente en todo momento. En una de las instituciones por ejemplo, se les pide a las autoridades de las sedes que los cursos empiezan unos tras otros, sin los 10 o 15 minutos de break que se acostumbran en la educación general y universitaria. Los profesores de matemática además son presionados para revisar contenidos en un tiempo muy acotado, lo cual choca con la realidad en el aula, con la capacidad que tienen los estudiantes para adquirir las competencias en un tiempo determinado y con la voluntad del profesor de apoyar a sus estudiantes.

\section{3. ¿ENSEÑANZA DE LA MATEMÁTICA AL SERVICIO DE LA ESPECIALIZACIÓN O DE LA FORMACIÓN GENERAL?}

En la mayoría de las instituciones los programas de estudio y las tareas que se deben realizar en el aula están altamente estandarizados. El diseño de estos programas se realiza a nivel central. Esta realidad se expresa sobre todo en las instituciones con presencia nacional y la estandarización se justifica como la única forma de asegurar que las competencias que se le entregan estudiantes en una sede, sean las mismas que se entregan en otra. Centralmente se definen los objetivos del ramo, los contenidos, se les entrega una estructura de clase a los profesores con propuestas de actividades y también se estandarizan las pruebas, quedando en teoría muy reducida la autonomía docente para adecuar los contenidos y las clases a la realidad de sus estudiantes.

Según los directivos de distintas instituciones ha existido una creciente tendencia por pasar de un enfoque tradicional a uno de formación por competencias, lo cual ha implicado una mayor preocupación de la institución por lo que se hace en el aula. Además, en el caso de las matemáticas, se aprovecha este énfasis en competencias para acercar la enseñanza a la especialización, o como muchos directivos la denominan, la "contextualización" de la matemática. La mayoría de los proyectos de innovación pedagógica apuntan en esa dirección y en muchos casos han provocado resistencia en los profesores.

Yo creo que el docente de matemática, primero, tiene que entender que las matemáticas son instrumentales en el ámbito nuestro y están al servicio, por lo tanto, de una disciplina específica, y por lo tanto tienen que ordenarse a esa disciplina, tienen que ajustarla a ese requerimiento y ojalá con ejemplos que sirvan para entender para qué me va a servir la matemática que me están enseñando en lo que yo voy a hacer (Directivo ESTP). 
En la percepción de directivos y profesores, a la mayoría de los estudiantes les interesa sólo lo directamente relacionado para sus necesidades laborales y en términos académicos sólo les interesa lo que va a ser evaluado, "lo que van a ver en la prueba". Existen por tanto, dos presiones para que el docente se concentre en una enseñanza al servicio de la especialización: la presión de los directivos y la de los estudiantes, las cuales chocan con muchos docentes que tienen como objetivo enseñar matemáticas para un objetivo mayor como brindar herramientas básicas para la vida laboral, fortalecer el pensamiento lógico e incluso formar ciudadanía.

[La institución] espera del docente que hagas tus clases bien, digamos, que tus evaluaciones que te hacen los alumnos sean buenas, que tengas todas las rutas formativas que estás desarrollando, es como súper formalito, el espacio de la formalidad. Pero yo creo que nosotros esperamos mucho más desde el área pedagógica, es decir, que lo que hacemos sea impactante para los chicos, sea significativo. Es decir que provoque significancia (Profesor, 15 años de experiencia).

\subsection{EL FANTASMA DE LA DESERCIÓN Y LA PRESIÓN POR LA APROBACIÓN}

El área de matemáticas es particularmente importante para la ESTP por su transversal presencia en las carreras impartidas, su condición de gatekeeper para la continuidad en la educación superior y por su relación con la deserción. Es por lo anterior que en la mayoría de las instituciones existen programas de innovación pedagógica en esta área junto con la incorporación de la matemática en los diferentes programas de retención.

Pensamos que la reprobación en matemática explica, todavía no tenemos el dato, no tenemos la investigación suficiente para poder decir en qué porcentaje, pero intuitivamente podríamos decir que la explica en un 50 o 60\%. Es determinante porque además en matemática en primer año, los alumnos reprueban del orden del 25-30\%, cercana a la retención de primer año (Directivo ESTP).

Derivado de la relación entre reprobación en matemáticas con la deserción estudiantil, hay docentes que sienten que la institución los presiona por aprobar a estudiantes y tener una política de evaluación a lo menos, flexible. Además, junto con la presión institucional, los docentes tienen internalizado que sus escalas de evaluación no pueden ser más rígidas porque de reprobar a estudiantes, la probabilidad que deserten es alta.

Si me pidieran cosas como aprobar para mantener la retención, aprobar por aprobar cuando yo sé que después viene un año súper grande, que nos ha pasado ya que hemos aprobado chicos en matemática que uno sabe que no tienen las competencias suficientes y que a la larga se transforman en una muerte anunciada, eso pasa y nadie quiere escuchar aquí. Yo en ese juego no voy a caer (Profesora, 10 años de experiencia).

La mayoría de los docentes parte desde una desconfianza a la capacidad de los estudiantes. En muchos casos los docentes comprenden e internalizan las exigencias de las instituciones, pero terminan redefiniéndolas en el camino, a propósito del nivel académico de los estudiantes. Por ejemplo, los profesores simplifican problemas o se detienen más 
Estudios Pedagógicos XLIV, N $^{\circ}$ 3: 235-252, 2018

INFLUENCIA DEL CONTEXTO INSTITUCIONAL EN EL TRABAJO DE LOS PROFESORES DE MATEMÁTICAS EN LA EDUCACIÓN SUPERIOR TÉCNICO-PROFESIONAL EN CHILE

tiempo del planificado en ciertos contenidos. Sin embargo, en la muestra de profesores participantes, encontramos varios episodios como el siguiente en el cual los profesores se sorprendían del resultado de sus estudiantes, abiertos a sus capacidades de aprender y resolver problemas de complejidad mayor a la esperada.

Facilitador: Claro, lo interesante es que acá sin tener modelo igual lo resolvieron. I profesor: Claro igual, igual lo hicieron. / profesora: Claro. / profesor: Sí poh, ellos no habían visto nada, entonces yo cuando planteé el, o sea, cuando puse el problema no quise hacerlo mayormente complejo porque yo dije estos chicos quizás no sepan hacer ni una potencia dije yo (Grupo de discusión $\mathrm{n}^{\circ} 7$ ).

Es el conocimiento del nivel de los estudiantes lo que le permite a los docentes manejar de mejor manera su trabajo en el aula. Quienes acomodan los mensajes institucionales tienen la capacidad de adaptar los ejercicios en función del nivel de sus estudiantes y por tanto, pese a no aplicar estrictamente lo que se les solicita, son capaces de lograr el objetivo de aprendizaje. Los docentes que hicieron sentido a partir de la acomodación, fueron capaces de adaptar "a la baja" y "al alza" en nivel de dificultad exigido en su clase.

\section{DISCUSIÓN}

Los resultados de la presente investigación refuerzan la necesidad de analizar críticamente la enseñanza de las matemáticas en la ESTP. Para esto, es importante conocer a los profesores y su trabajo dentro y fuera del aula. Los profesores de estas instituciones no van solo a verter conocimiento a sus estudiantes. Su labor docente es mucho más compleja porque tienen que atender a las particularidades de sus estudiantes, que tienen no tienen solo necesidades pedagógicas, sino que incluso afectivas. En la muestra de profesores participantes observamos que ellos tienen un alto compromiso con sus estudiantes porque tienen vocación de profesores. Están involucrados en el proceso de aprendizaje de sus estudiantes y eso debe ser relevado por la institución. Aumentar las horas en los contratos de los docentes -o disminuir la proporción de las horas de clases en relación a las horas no lectivas- puede tener efectos positivos en la tasas de retención en las instituciones y en el grado de aprobación en matemáticas de los estudiantes.

Los contratos a profesores de matemáticas por áreas diferentes limita la posibilidad del trabajo mancomunado entre docentes y de desarrollar una mayor colegialidad. La mayoría de los profesores participantes plantearon que la conversación entre docentes, el intercambio de experiencias e incluso el desarrollo de una identidad de "profes de matemáticas", es un factor que posibilitaría mejoras en el trabajo en el aula, por lo que una forma de contratación que fomentara dichas prácticas, podría facilitar estas externalidades positivas.

Una de las obligaciones que mayormente limita el trabajo docente, es la permanente exigencia de eficiencia en el uso de los tiempos. La no existencia de espacios entre clases y la compresión de horas pedagógicas para un mismo ramo no sólo deriva en que los profesores no puedan implementar lo planificado, sino que también, paradójicamente, en un uso ineficiente del tiempo. La exigencia por la eficiencia en el uso del tiempo es uno de los principales motivos por los cuales los docentes rechazan los mensajes de su entorno. 
Dotar un sentido de realidad al uso de los tiempos por tanto, posibilitaría una mejor implementación de las diferentes políticas pedagógicas de las instituciones en el aula.

En la definición de contenidos, resultados de aprendizaje, descriptores o maletas pedagógicas es necesario un mayor intercambio entre la institución con el estamento docente. Una de las principales críticas de los profesores apunta en esta dirección: el nivel central de la institución planifica sin una conversación con lo que pasa en el aula. Junto con este mayor diálogo, podría ser positivo que la institución les entregara más autonomía a los profesores en relación a lo que sucede en la sala de clases. Es necesario resguardar la necesidad de homogenizar los contenidos para asegurar que quienes egresan de cada una de las sedes tengan las mismas competencias, pero la realidad indica que cada curso tiene su particularidad y depende de esa particularidad los ritmos en que es necesario avanzar. Mayores libertades para que los docentes puedan planificar sus clases (acompañado por contratos con horas que lo permitan) aumentaría el nivel de acomodación ante las exigencias de la institución.

La necesidad de dotar de mayor margen a los docentes sobre lo que sucede en la sala de clases, también tiene que aplicarse en las formas de evaluación a los estudiantes. Una de las principales causas de que se genere una respuesta simbólica a las demandas de la institución (Coburn, 2004) es que los docentes tienen que cumplir con lo que va a ser evaluado centralmente. Cuando el foco está puesto en el aprendizaje y en el "aprender haciendo", no es positivo que las clases estén limitadas totalmente a lo que "se va a ver en la prueba".

En general las instituciones le brindan a los docentes una importante cantidad de recursos pedagógicos (planificaciones de clases, recursos tecnológicos, capacitaciones, entre otras) para el desarrollo de sus clases. El problema es que los profesores suelen echar mano a sus propios recursos para estos fines, generando una dinámica de creación de estructuras paralelas, que se potencia además por las propias limitaciones disciplinares de las y los docentes y por no contar con el tiempo suficiente para reflexionar sobre los recursos a su disposición.

\section{CONCLUSIÓN}

Los resultados de la investigación ponen en evidencia que gran parte de las obligaciones institucionales no están pensadas para apoyar el trabajo del docente y en muchos casos se transforman en trabas para un buen trabajo en el aula. La lejanía de los órganos directivos percibida por los profesores con la realidad en el aula hace que las políticas pedagógicas y administrativas como el uso de los tiempos, vayan a contramano de lo que ven los docentes en su día a día; la realidad profesionalizante y dirigida a la especialización de la ESTP choca con la voluntad del docente de apoyar integralmente a sus estudiantes y la presión por evitar la deserción se aborda de forma superficial (flexibilizando criterios de evaluación) y no buscando mejorar el proceso educativo a través del mejoramiento continuo de la labor docente o brindándole herramientas para que puedan contextualizar los contenidos a la realidad del aula. Parte importante de las limitaciones docentes producto de las obligaciones institucionales se puede reducir al hecho de que las instituciones no diferencian el trabajo de los profesores de matemáticas, con la de sus pares de especialidad.

Las limitaciones de la presente investigación abren la puerta a estudios más profundos sobre la realidad de la ESTP en Chile. Hay una primera limitación derivada de la muestra: 
Estudios Pedagógicos XLIV, N $^{\circ}$ 3: 235-252, 2018

INFLUENCIA DEL CONTEXTO INSTITUCIONAL EN EL TRABAJO DE LOS PROFESORES DE MATEMÁTICAS EN LA EDUCACIÓN SUPERIOR TÉCNICO-PROFESIONAL EN CHILE

se abarcó a las instituciones con mayor matrícula (lo que abarca a la gran mayoría de estudiantes de estas instituciones), pero se dejó fuera del análisis a las instituciones de menor tamaño, las cuales tienen sus propias particularidades. Junto con lo anterior, sería importante contrastar la realidad de los docentes de matemáticas con sus pares de especialidad.

Los resultados indican que los profesores de matemáticas están involucrados en el proceso de enseñanza y están comprometidos por mejorarlo. Es por lo anterior que es importante realizar futuras investigaciones que analicen cómo las instituciones de la ESTP fomentan la capacidad de agencia de los docentes, así como también de que forma la limitan.

Los desafíos que tiene Chile en relación a la ESTP son enormes. Esta investigación tiene por objetivo contribuir a discutir sobre estos desafíos poniendo énfasis en lo que pasa en el aula y en el trabajo de los profesores. Queda en manos de los profesores de estas instituciones, en los directivos y en quienes construyen políticas públicas en torno a la ESTP que esta discusión se siga profundizando con el objetivo de tener una ESTP que esté a la altura de los desafíos del Chile del siglo XXI.

\section{REFERENCIAS BIBLIOGRÁFICAS}

Ball, D. L., Lubienski, S. \& Mewborn, T. (2001). Research on teaching mathematics: The unsolved problem of teachers' mathematical knowledge. In V. Richardson (Ed.), Handbook of research on teaching (4th ed., pp. 433-456). New York, NY: Macmillan.

Berger, P. \& Luckmann, T. (1966). The Social Construction of Reality. New York, NY: Doubleday Anchor.

Blair, R., Kirkman, E. \& Maxwell, J. (2013). Statistical abstract of undergraduate programs in the mathematical sciences in the United States. Fall 2010 CBMS Survey. Washington DC: AMS.

Braun, V. \& Clarke, V. (2012). Thematic Analysis. In H. Cooper, Handbook of Research Methods in Psychology (2, pp. 57-71). American Psychological Association.

Carpenter, T. P., Fennema, E. \& Franke, M. L. (1996). Cognitively guided instruction: A knowledge base for reform in primary mathematics instruction. The Elementary School Journal, 97(1), $3-20$.

Carpenter, T. P., Fennema, E., Franke, M. L., Levi, L. \& Empson, S. B. (1999). Children's Mathematics: Cognitively Guided Instruction. Portsmouth, NH: Heinemann.

Chazan, D. \& Herbst, P. (2012). Animations of Classroom Interaction: Expanding the Boundaries of Video Records of Practice. Teacher's College Record, 114(3), 1-34.

Chazan, D., Herbst, P. \& Clark, L. (2016). Research on the Teaching of Mathematics: A Call to Theorize the Role of Society and Schooling in Mathematics Instruction. In C. Bell, \& D. Gitomer (Eds.), Handbook of research on teaching (5a ed., pp. 1039-1097). Washington DC: American Educational Research Association.

CNED (2017). INDICES-CNED. Consejo Nacional de Educación. Recuperado el 20 de diciembre del 2017 desde: www.cned.cl/public/Secciones/SeccionIndicesPostulantes/Indices_Sistema. aspx

Coburn, C. E. (2004). Beyond Decoupling: Rethinking the Relationship between the Institutional Enviroment and the Classroom. Sociology of Education, 77(3), 211-244.

Cohen, D. K., Raudenbush, S. W. \& Ball, D. L. (2003). Resources, Instruction and Research. Educational Evaluation and Policy Analysis, 25(2), 119-142.

Cuban, L. (1984). How Teachers Taught: Constancy and Change in American Classrooms 1890- 
1980 (2a ed.). New York, NY: Longman.

Deal, T. E. \& Celloti, L. D. (1980). How Much Influence Do (and Can) Educational Administrators Have on Classrooms? Phi Delta Kappan, 61, 471-473.

Driscoll, M. E. (1995). We Have the Right to Be Different: Educational Community Through a Neoinstitutional Lens. Journal of Education Policy, 10(1-6), 55-68.

EEPA. (1990). Educational Evaluation and Policy Analysis, 12(1), 253-353.

Espejo, Alejandro. (2016). La Educación Técnico-profesional en el Futuro. Estudios Sociales, 124, 161-178.

Herbst, P. \& Chazan, D. (2011). Research on practical rationality: Studying the justifications of actions in mathematics teaching. The Mathematic Enthusiast, 8(3), 405-462.

Hill, H. C., Ball, D. L. \& Schilling, S. G. (2008). Unpacking pedagogical content knowledge: Conceptualizing and measuring teachers' topic-specific knowledge of students. Journal of Research in Mathematics Education, 34(4), 372-400.

Hodara, M. \& Jaggars, S. (2014). An examination of the impact of accelerating community college students' progression through developmental education. The Journal of Higher Education, 85(2), 246-276.

Hora, M. T. \& Ferrare, J. J. (2013). Instructional systems of practice: A multidimensional analysis of math undergraduate course planning and classroom teaching. Journal of the Learning Sciences, 22(2), 212-257.

Horn, I. S. \& Kane, B. D. (2015). Opportunities for Professional Learning in Mathematics Teacher Workgroup Conversations: Relationships to Instructional Expertise. Journal of the Learning Sciences, 24(3), 373-418.

Kim, J., Kim, J., DesJardins, S. \& McCall, B. (2015). Taking Algebra II in High School: Does it Increase College Access and Success? Journal of Higher Education, 86(4), 628-662.

Kunter, M. \& Voss, T. (2013). The Model of Instructional Quality in COACTIV: A Multicriteria Analysis. In M. Kunter et al. (Eds.), Cognitive activation in the mathematics classroom and professional competence of teachers: Results from the COACTIV Project (pp. 97-124). New York, NY: Springer.

Lande, E. \& Mesa, V. (2016). Instructional decision making and agency of community college mathematics faculty. ZDM, 48(1), 199-212.

Lattuca, L. R. \& Stark, J. S. (2009). Shaping the college curriculum: Academic plans in context. San Francisco, CA: Jossey-Bass.

Leindhardt, G. (1989). Math Lessons: A contrast of Novice and Expert Competence. Journal for Research in Mathematics Education, 20(1), 52-75.

Leindhardt, G. \& Greeno, J. G. (1986). The Cognitive Skill of Teaching. Journal of Educational Psicology, 78, 75-95.

Malen, B. \& Oganawa, R. T. (1988). Professional-Patron Influence on Site-Based Governance Councils: A Confounding Case Study. Educational Evaluation and Policy Analysis, 10, 251-270.

Malen, B., Oganawa, R. T. \& Kranz, J. (1990). What Do We Know about School-Based Management? A Case Study of the Literature-A Call for Research. En W. H. Clune \& J. F. Witte, (Eds.) Choice and Control in American Education, vol. 2, 289-342.

Mason, J. (2016). Perception, interpretation and decision making: understanding gaps between competence and performance-a commentary. ZDM, 48(1-2), 219-226.

Melguizo, T., Kosiewicz, H., Prather, G. \& Bos, J. (2014). How are community colleges assessed and placed in developmental math? The Journal of Higher Education, 85(5), 691-772.

Menéndez, Jorge (2017). La Educación Superior Técnico Profesional (ESTP), evolución y perspectiva de su desarrollo en Chile. Estudios Sociales, 125, 105-134.

Merriam, S. B. (2009). Qualitative Research: A Guide to Design and Implementation. San Francisco, CA: Jossey-Bass.

Mesa, V., Celis, S. \& Lande, E. (2014). Teaching approaches of community college mathematics 
Estudios Pedagógicos XLIV, N $^{\circ}$ 3: 235-252, 2018

INFLUENCIA DEL CONTEXTO INSTITUCIONAL EN EL TRABAJO DE LOS PROFESORES DE MATEMÁTICAS EN LA EDUCACIÓN SUPERIOR TÉCNICO-PROFESIONAL EN CHILE

faculty: Do they relate to classroom practices? American Educational Research Journal, 51(1), 117-151.

Metz, M. H. (1989). Real School: A Universal Drama Amid Disparate Experience. In D. E. Mitchell \& M. E. Goertz, Education Politics for the New Century (pp. 75-91). New York, NY: Falmer Press.

Meyer, J. \& Rowan, B. (1978). The Structure of Educational Organizations. San Francisco, CA: Jossey-Bass.

Meyer, J. \& Rowan, B. (1977). Institutionalized Organizations: Formal Structure as Myth and Ceremony. American Journal of Sociology, 83, 340-363.

MINEDUC. (2012). Educación Técnica Profesional en Chile. Antecedentes y claves de diagnóstico. División de Planificación y Presupuesto, Ministerio de Educación, Gobierno de Chile.

Paredes, R. \& Sevilla, M.P. (2017). Reforma de la educación superior técnico profesional. Estudios sociales, $125,17-42$.

Rowan, B. \& Miskel, C. C. (1999). Institutional Theory and the Study of Educational Organizations. In J. Murphy, \& K. S. Louis, Handbook of Research on Educational Administration (2a ed., pp. 359-384). San Francisco, CA: Jossey-Bass.

Sanhueza, J. M. (2015). Instituciones de Educación Superior en un contexto de mercado: el caso de la Educación Técnico-Profesional en Chile (Tesis no publicada). Universidad de Chile.

Schoenfeld, A. H. (2016). Making sense of teaching. ZDM, 48(1), 239-246.

Schoenfeld, A. H. (1985). Mathematical problem solving. Orlando, FL: Academic Press.

Sfard, A. (2001). There is more to discourse than meets the ears: Looking at thinking as communicating to learn more about mathematical learning. Educational Studies in Mathematics, 46, 13-47.

SIES. (2018a). Compendio histórico de educación superior. Santiago: Ministerio de Educación. Recuperado el 20 de junio del 2018 desde: http://www.mifuturo.cl/index.php/estudios/ estructura-compendio

SIES. (2018b). Informe Matrícula 2018. Santiago: Ministerio de Educación. Recuperado el 20 de junio del 2018 desde: http://www.mifuturo.cl/index.php/informes-sies/matriculados

SIES. (2016a). Informe de retención de ler año de pregrado cohortes 2011-2015. Santiago: Ministerio de Educación. Recuperado el 15 de diciembre del 2017 desde: https://biblioteca. digital.gob.cl/bitstream/handle/123456789/693/informe\%20retencion_sies_2016. pdf? sequence $=1 \&$ is Allowed $=\mathrm{y}$

SIES. (2016b). Retención del Primer Año en Educación Superior. Santiago: Ministerio de Educación. Recuperado el 15 de diciembre del 2017 desde: http://www.mifuturo.cl/index.php/informessies/retencion-de-pregrado

SIES. (2014). Panorama de la Educación Superior en Chile. División de Educación Superior. Santiago: Ministerio de Educación.

Steinbring, H. (2005). Analyzing mathematical teaching-learning situations - the interplay of communicational and epistemological constraints. Educational Studies in Mathematics, 59(13), 313-324.

Talbert, J. E. \& McLaughlin, M. W. (1994). Teacher Professionalism in Local School Contexts. American Journal of Education, 102, 123-153.

Weick, K. E. (1995). Sensemaking in Organizations. Oaks, CA: Sage.

Weick, K. E., Sutcliffe, K. M. \& Obstfeld, D. (2005). Organizing and the Process of Sensemaking. Organization Science, 16(4), 409-421.

Yackel, E. \& Cobb, P. (1996). Sociomathematical norms, argumentation, and autonomy in mathematics. Journal for Research in Mathematics Education, 27, 458-477. 